\title{
ESTUDIO MORFOLÓGICO COMPARADO DE VERRUCARIA MAURA Y V. AMPHIBIA EN LA COSTA VASCA
}

\author{
Gustavo RENOBALES \& Rosario NOYA
}

\begin{abstract}
RESUMEN: Se estudia la variabilidad que presentan las poblaciones de Verrucaria maura y $V$. amphibia de la costa vasca. Aparte de las formas típicas, se reconocen tres fenotipos diferentes, uno de ellos perteneciente a $V$. amphibia y los otros dos a V. maura. La principal variabilidad que presenta $V$. maura se relaciona con la altura a la que se encuentra en la costa. Los caracteres más útiles para la discriminación entre las dos especies son la forma del borde del talo y la anchura de las esporas.
\end{abstract}

Palabras clave: Morfología, variabilidad, esporas, Verrucaria.

ABSTRACT: Morphological variability in Verrucaria maura and V.amphibia is studied from populations living in the Basque Coast ( $\mathrm{N}$-Spain). Five different phenotypes are recognized, two of them belonging to V. amphibia (typical and type «a»1), while the other three belong to V. maura (typical and types «m»1 and «m»2). Their morphology is summarized and compared in tables 1 and 2 . Their macroscopic habit is shown in figs. 1-5.

The most constant characters, hence the most suitable to distinguish between the two species, are the border of the thallus, wich is abrupt and without hypothallus in V. amphibia while it is thin and has a clear hypothallus in V. maura, and the size of spores, constantly broader in V. maura. All other morphological characters are far more variable and less useful for discrimination. The main variability found in V. maura is related with the relative level occupied by the specimens in the coast, while that found in V. amphibia is probably related with the degree of insolation.

One of the phenotypes of V. maura, t. «m» 2 , is in uncertain position for the moment, as it appears to be intermediate between the two species.

Key words: Morphology, variability, spores, Verrucaria.

\section{INTRODUCCIÓN}

La discriminación taxonómica entre las especies de pirenolíquenes costeros Verrucaria maura Wahlenb. y V. amphibia R. Clem. suele ser problemática debido a su relativa semejanza morfológica y ecológica. Puesto que ambas pueden encontrarse en ambientes similares (horizonte costero litoral superior, Fletcher, 1973), en muchas ocasiones se encuentran formando mosaicos, a veces tan intrincados que es difícil 
determinar las partes que corresponden a una especie o a la otra. El problema se agrava porque, si bien la morfología «típica» de ambos taxones es suficientemente característica, abundan los ejemplares cuya morfología se desvía bastante de ésta y cuya adscripción a uno u otro es muy difícil sin información suficiente acerca de su variabilidad.

En este trabajo planteamos precisamente el análisis de la variabilidad encontrada en ambas especies después del estudio de un buen número de ejemplares recogidos en la Costa Vasca en los últimos años. Los caracteres que hemos considerado significativos han sido los caracteres morfológicos tradicionales: color del talo, grado de areolamiento, borde del talo, forma y posición de los ascomas, dimensiones esporales, etc. No se han estudiado ni la química ni la anatomía. El carácter que hemos denominado ornamentación del talo (tabla 1) se refiere a la distribución que presentan las partes carbonáceas en la superficie del mismo. Mientras que en las formas más típicas de $V$. maura la superficie de las aréolas es prácticamente lisa y de color uniformemente oscuro, en las formas típicas de V. amphibia la superficie del talo presenta en su parte periférica netas costillas salientes de color negro y disposición más o menos radial. Entre ambos extremos existen muchos tipos intermedios. Es un carácter que depende del grosor del talo y que hay que evaluar siempre con mucho cuidado.

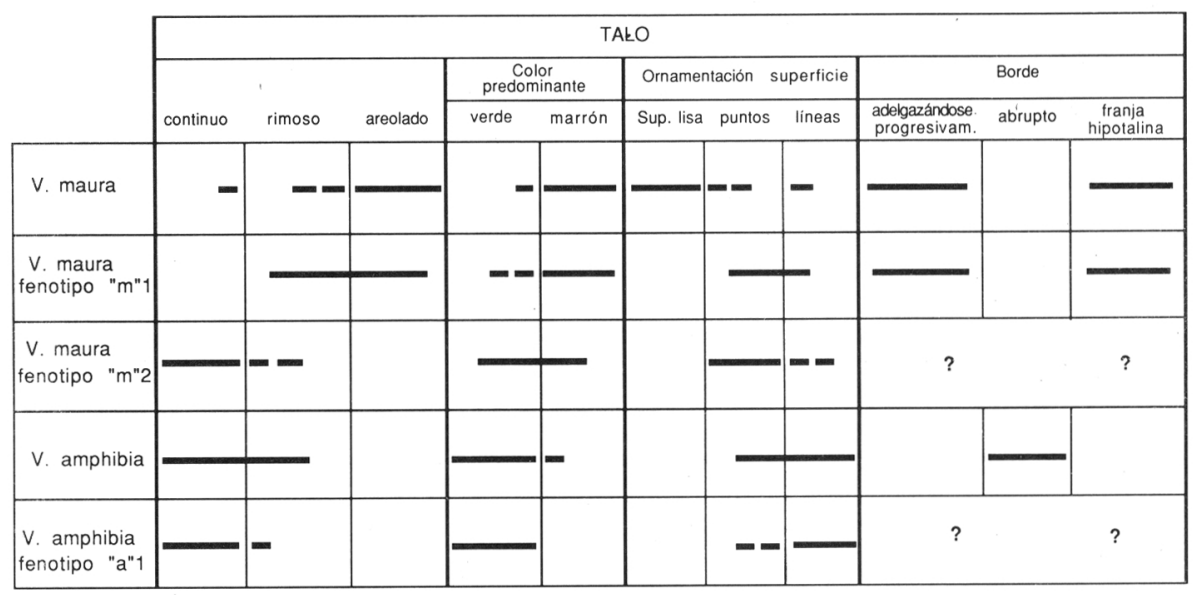

Tabla 1. Cuadro comparativo entre las formas estudiadas de V. maura y V. amphibia. Caracteres morfológicos talinos.

\section{RESULTADOS Y DISCUSIÓN}

Para la representación de la variabilidad en ambas especies hemos agrupado los especímenes estudiados en 5 fenotipos que corresponden a las formas que pueden reconocerse y diferenciarse macroscópicamente. Estas son: V. maura típica y fenotipos 
«m»1 y «m»2, y $V$. amphibia típica y fenotipo «a»1. Puesto que la variabilidad que presentan ambas especies depende directamente de las condiciones ecológicas no creemos necesario conceder rango taxonómico a tales tipos, aunque el caso del fenotipo «m»2 es el más dudoso. La morfología de cada uno de ellos se resume en las tablas 1 y 2, y las dimensiones esporales se representan y comparan en los gráficos 1 y 2. En las figuras 1-5 se representa el aspecto macroscópico de cada uno.

$V$. maura presenta una variabilidad acusada, como ha sido reconocido por algunos autores, como Fletcher (1975). Clauzade \& Roux (1985) también lo reconocen de forma implícita, al englobar en este taxon otros considerados independientes por Zschacke (1933-34). La principal variabilidad que nosotros hemos encontrado en este taxon está relacionada con el nivel que ocupan los ejemplares en el tramo costero. El fenotipo «m»1 se encuentra en los niveles inferiores del horizonte supralitoral, mientras que la morfología típica (fig. 1) aparece sobre todo en los niveles medios y superiores.
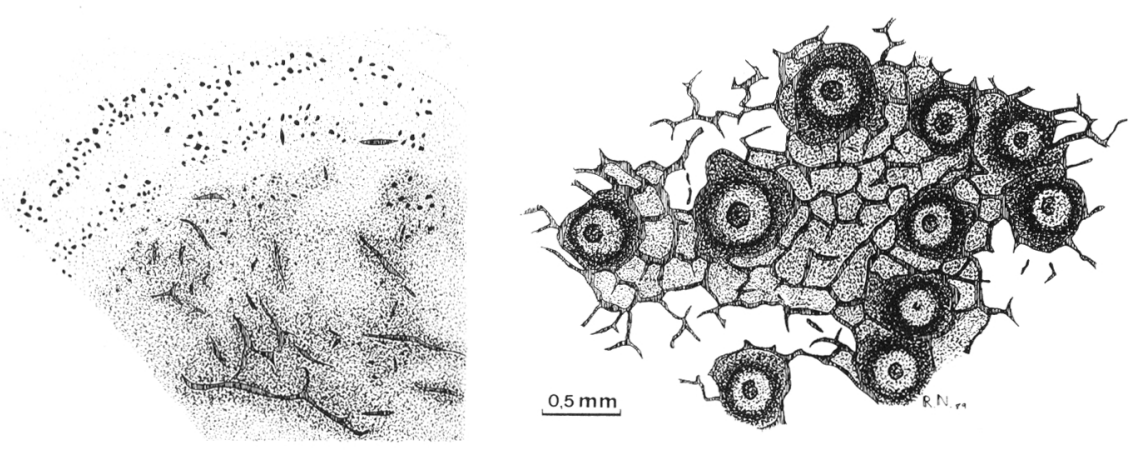

Fig. 1. Aspecto macroscópico típico de V. maura.

En el tipo morfológico «m»1 (fig. 2) el talo es menos areolado y con areolas menores de $0.3 \mathrm{~mm}$ de anchura, lo que le da un aspecto casi continuo a simple vista. La superficie del talo es rugosa debido a que las partes carbonáceas del mismo sobresalen en forma de pequeños puntos o microrrugosidades, y en ocasiones llegan a ser cortas líneas, a veces muy netas y dispuestas radialmente en la periferia del talo. Los ascomas tienen una marcada tendencia a ser semiinmersos en estos ejemplares, y de contorno a veces muy irregular lo que contrasta notablemente con los ascomas regulares e inmersos de las formas típicas de V. maura, casi siempre completamente recubiertos por el talo. La morfología del fenotipo «m»1 difiere bastante de la típica de $V$. maura y puede llegar a aproximarse bastante en algunos aspectos a $V$. amphibia. La posibilidad de confusión entre ambas se acentúa por el hecho de que ambas suelen coincidir en el mismo nivel costero, y aparecen muy frecuentemente en mosaico. Las dimensiones esporales (gráf. 1 y 2) y la forma del borde del talo son los caracteres discriminantes más seguros. 


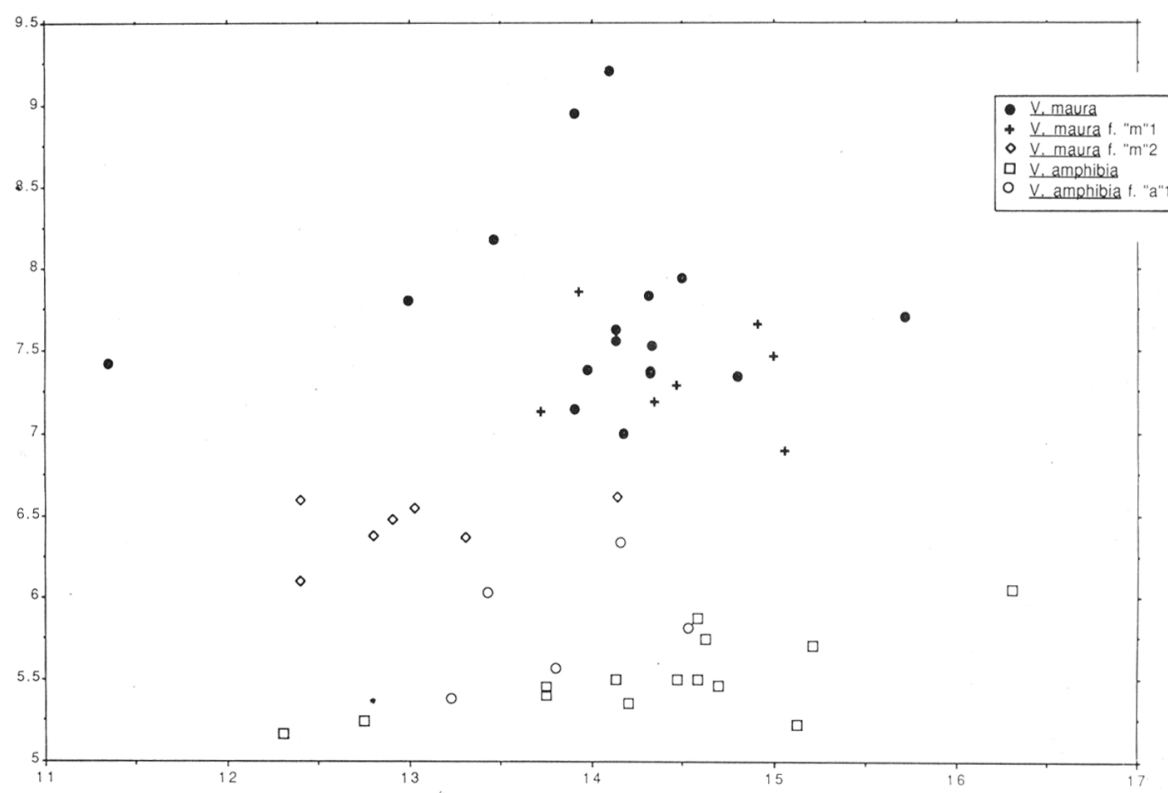

Gráf. 1. Representación de las medidas de las dimensiones esporales $(\mu \mathrm{m})$ para cada uno de los ejemplares estudiados.

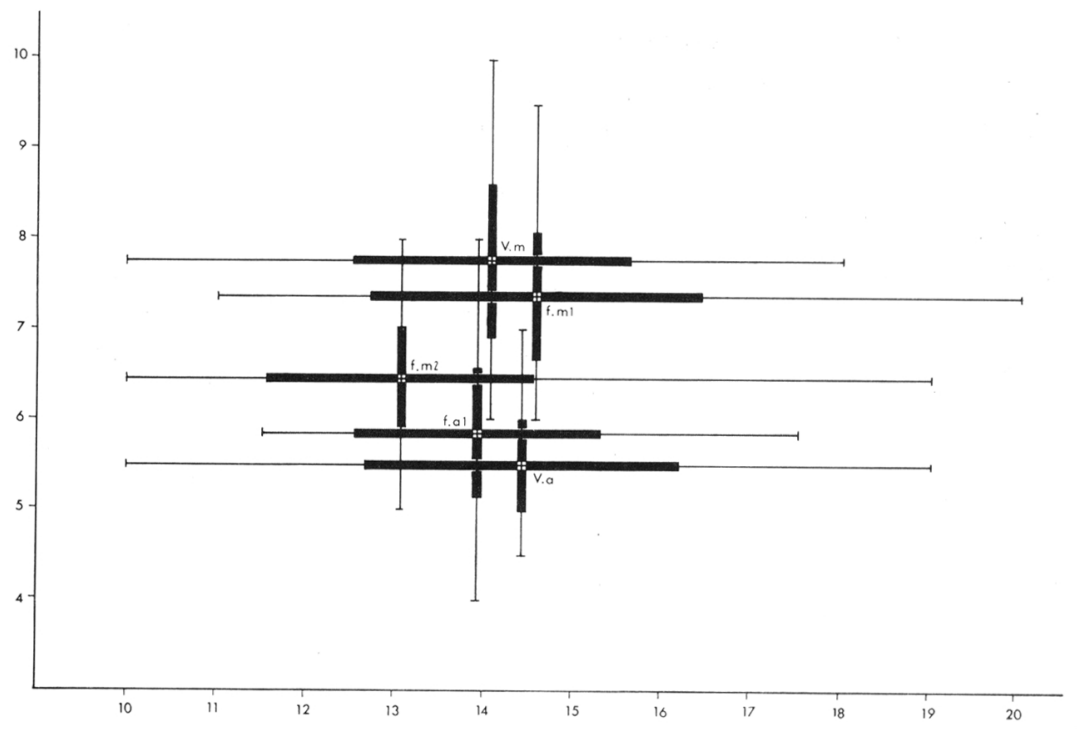

Gráf. 2. Dimensiones esporales (medias, valores extremos y desviación típica) para cada uno. 

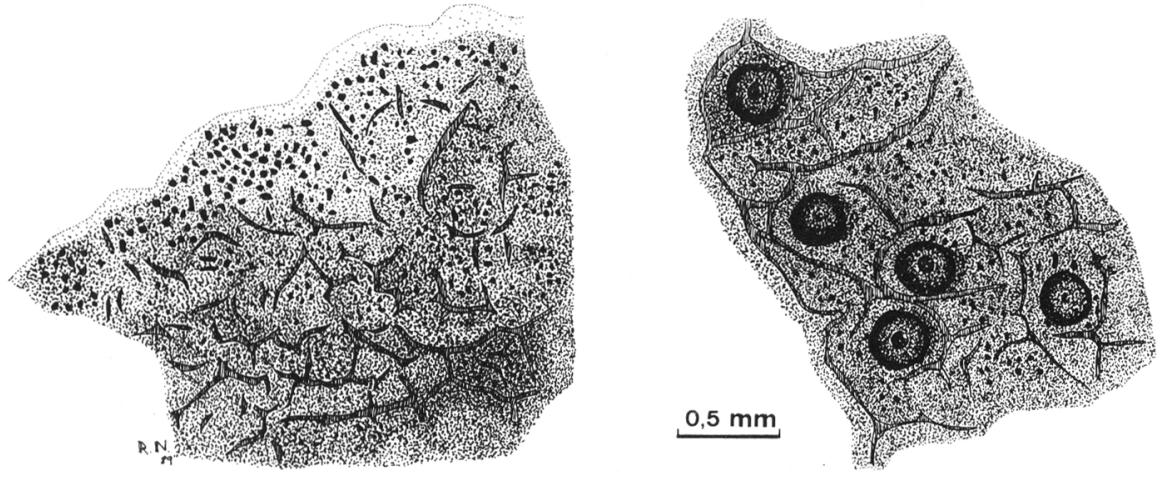

Fig. 2. Aspecto macroscópico de V. maura fenotipo «m»1.

En V. maura el talo se adelgaza progresivamente hacia la periferia, y presenta casi siempre una franja hipotalina de color crema claro que puede estar más o menos desarrollada. Es especialmente visible cuando el sustrato es liso, pero no es fácil de observar en mosaicos muy densos. En V. amphibia (fig. 4) el talo no se adelgaza hacia la periferia, sino más bien tiende a engordar, y el borde es abrupto. No hay franja hipotalina y sí una línea periférica oscura, bien visible en la mayoría de los casos.

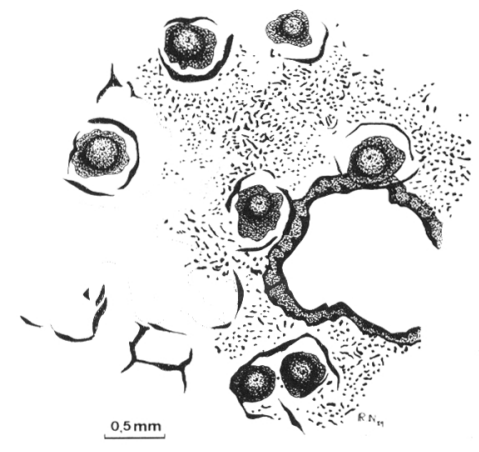

Fig. 3. Aspecto macroscópico de V. maura fenotipo «m»2.

$V$. amphibia se encuentra dentro de un rango altitudinal más estrecho que $V$. maura (Fletcher, 1973) lo que explica que la variabilidad de este taxon con respecto a este factor sea inapreciable. El fenotipo «a»1 que reconocemos corresponde más bien a una forma esciáfila de esta especie ya que șiempre se encuentra en biotopos protegidos. Dicho fenotipo (fig. 5) es bastante característico por su talo delgado, 
continuo, de color verde, con conspicuas líneas negras salientes que tienen tendencia a disponerse ordenadamente. Los ascomas, sésiles y bastante irregulares parecen entrar dentro del rango de variabilidad morfológica de $V$. amphibia así como las medidas de las esporas. La peculiar morfología del talo puede ser debida a la falta de luz (Fletcher, 1975). En estos ejemplares no hemos podido observar el borde del talo ya que suelen ser bastante fragmentarios. Este fenotipo morfológico se encuentra frecuentemente asociado a $V$. striatula Wahlenb.
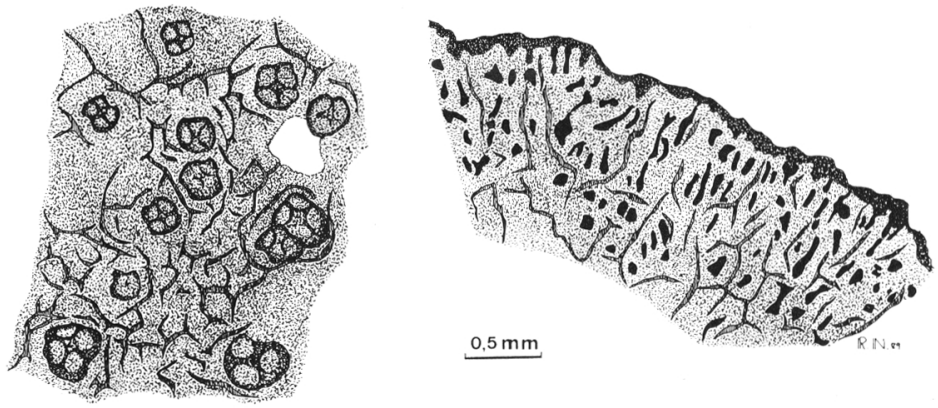

Fig. 4. Aspecto macroscópico de V. amphibia.

El fenotipo «m»2 (fig. 3) es de interpretación menos segura. El talo es muy delgado y tiende a crecer formando manchas extensas y mal definidas. Es de color verde o marrón, con numerosos puntos negros, que algunas veces llegan a formar líneas. No hemos podido observar el borde. Los ascomas son hemisféricos y bastante regulares, casi siempre bastante recubiertos por el talo en el tercio inferior. Por su morfología, y sobre todo por la forma de los ascomas, consideramos este fenotipo como una forma de $V$. maura. Sin embargo las esporas son constantemente más estrechas que las de esa especie (gráf. 1 y 2) y se introducen bastante en el rango de variabilidad que presentan V. amphibia y su forma «a»1, aún sin llegar a ser iguales a éstas. Puesto que por el momento no puede ser concluyente sobre la categoría taxonómica que puedan tener estos ejemplares preferimos asignarlos provisionalmente a un tipo morfológico de V. maura.

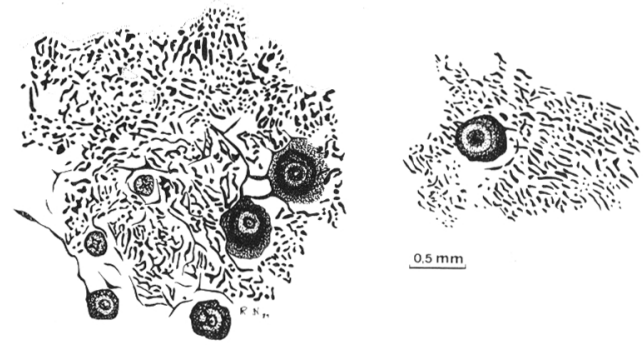

Fig. 5. Aspecto macroscópico de V. amphibia fenotipo «a»1. 


\begin{tabular}{|c|c|c|c|c|c|c|c|c|}
\hline & \multicolumn{8}{|c|}{ ASCOMA } \\
\hline & \multicolumn{3}{|c|}{ Posición } & \multicolumn{3}{|c|}{ Forma } & \multicolumn{2}{|c|}{ Esporas (v. Gráfs. 1 y 2) } \\
\hline & inmerso & semiinmerso & sésil & hemistérico & irregular & crenulado & Dimensiones & Valores medios \\
\hline V. maura & & & & & & & $10.17(18) \times 6.10$ & $\begin{array}{l}\text { L: } 14.06 \pm 1.56 \\
\text { A: } 7.74 \pm 0.84\end{array}$ \\
\hline $\begin{array}{l}\text { V. maura } \\
\text { fenotipo "m"1 }\end{array}$ & & & & & $=$ & & $11-17(20) \times 6-8(9)$ & $\begin{array}{l}\text { L: } 14.58 \pm 1.85 \\
\text { A: } 7.34 \pm 0.70\end{array}$ \\
\hline $\begin{array}{l}\text { V. maura } \\
\text { fenotipo "m"2 }\end{array}$ & & & & & & & $(10) 11 \cdot 15(19) \times 5 \cdot 7(8)$ & $\begin{array}{l}\text { L: } 13.06 \pm 1.5 \\
\text { A: } 6.45 \pm 0.59\end{array}$ \\
\hline V. amphibia & & & & & & & $(10) 12.17(19) \times 4.5-6(7)$ & $\begin{array}{l}\text { L: } 14.38 \pm 1.77 \\
\text { A: } 5.51 \pm 0.54\end{array}$ \\
\hline $\begin{array}{l}\text { V. amphibia } \\
\text { fenotipo "a"1 }\end{array}$ & & & & & & & $11 \cdot 16(18) \times 4 \cdot 7(8)$ & $\begin{array}{l}\text { L: } 13.88 \pm 1.38 \\
\text { A: } 5.87 \pm 0.73\end{array}$ \\
\hline
\end{tabular}

Tabla 2. Cuadro comparativo entre las formas estudiadas de V. maura y V. amphibia. Caracteres morfológicos ascomáticos.

\section{CONCLUSIONES}

La principal variabilidad detectada en V. maura está relacionada con la posición que ocupa en la costa. Los ejemplares menos típicos aparecen en los niveles inferiores, frecuentemente junto con V. amphibia con quien pueden confundirse fácilmente. Esta segunda especie es menos variable según este factor pues su rango altitudinal en la costa es también menor.

Caracteres morfológicos tales como el grado de areolamiento del talo, el modelo de distribución de tejidos carbonáceos en el talo en vista superficiảl o la forma macroscópica de los ascomas son altamente variables, y por tanto los más interesantes para la discriminación taxonómica son la anchura de las esporas y el borde del talo, delgado y con hipotalo, o bien abrupto y sin hipotalo.

Por último, existe un fenotipo, aquí denominado fenotipo «m» 2 , que presenta caracteres intermedios entre ambas especies. Provisionalmente lo incluimos dentro de la variabilidad de V. maura. 


\section{BIBLIOGRAFÍA}

CLAUZADE, G. \& CI. ROUX -1985- Likenoj de okcidenta europo. Ilustrita determinlibro. Bull. Soc. Bot. du Centre-Ouest, nouv. sér. num. spéc. 7:1-893.

FLETCHER, A. -1973- The ecology of marine (littoral) lichens on some rocky shores of Anglesey. Lichenologist 5:368-400.

FLETCHER, A. -1975- Key for the identification of British marine and maritime lichens. I. Siliceous rocky shore species. Lichenologist 7:1-52.

ZSCHACKE, H. -1933-34- Epigloeaceae, Verrucariaceae und Dermatocarpaceae. Rabenh. Krypt.Fl. 9 (1): 44-695.

(Aceptado para su publicación en Junio de 1.990 )

Dirección de los autores: Departamento de Biología Vegetal y Ecología. Universidad del País Vasco. Ap. 644. 48080 Bilbao 